\title{
The blue plume population in dwarf spheroidal galaxies
}

\section{Genuine blue stragglers or young stellar population?`}

\author{
Y. Momany ${ }^{1}$, E. V. Held ${ }^{1}$, I. Saviane ${ }^{2}$, S. Zaggia ${ }^{1}$, L. Rizzi ${ }^{3}$, and M. Gullieuszik ${ }^{1}$ \\ 1 INAF: Osservatorio Astronomico di Padova, vicolo dell'Osservatorio 5, 35122 Padova, Italy \\ e-mail: [yazan.almomany; enrico.held;marco.gullieuszik; simone.zaggia]@oapd.inaf.it \\ 2 European Southern Observatory, A. de Cordova 3107, Santiago, Chile \\ e-mail: isaviane@eso.org \\ 3 Institute for Astronomy, 2680 Woodlawn Drive, Honolulu, HI 96822, USA \\ e-mail: rizzi@ifa.hawaii.edu
}

Received 22 December 2006 / Accepted 3 April 2007

ABSTRACT

\begin{abstract}
Aims. Blue stragglers (BSS) in the Milky Way field and globular/open clusters are thought to be the product of either primordial or collisional binary systems. In the context of dwarf spheroidal galaxies it is hard to firmly disentangle a genuine BSS population from young main sequence (MS) stars tracing a $\sim 1-2$ Gyr old star forming episode.

Methods. Assuming that their blue plume populations are made of BSS, we estimate the BSS frequency ( $F_{\mathrm{HB}}^{\mathrm{BSS}}$; as normalized to the horizontal branch star counts) for 8 Local Group non star-forming dwarf galaxies, using a compilation of ground and space based photometry.

Results. (i) The BSS frequency in dwarf galaxies, at any given $M_{V}$, is always higher than that in globular clusters of similar luminosities; (ii) the BSS frequency for the lowest luminosity dwarf galaxies is in excellent agreement with that observed in the Milky Way halo and open clusters; and most interestingly (iii) derive a statistically significant $F_{\mathrm{HB}}^{\mathrm{BSS}}-M_{V}$ anti-correlation for dwarf galaxies, similar to that observed in globular clusters.

Conclusions. The low density, almost collision-less, environments of our dwarf galaxy sample allow us to infer (i) their very low dynamical evolution; (ii) a negligible production of collisional BSS; and consequently (iii) that their blue plumes are mainly made of primordial binaries. The dwarf galaxies $F_{\mathrm{HB}}^{\mathrm{BSS}}-M_{V}$ anti-correlation can be used as a discriminator: galaxies obeying the anti-correlation are more likely to possess genuine primordial BSS rather than young main sequence stars.
\end{abstract}

Key words. stars: galaxies: dwarf - blue stragglers - galaxies: stellar content - stars: Hertzspring-Russell (HR) and C-M diagrams galaxies: Local Group - galaxies: photometry

\section{Introduction}

In the context of Galactic Globular clusters studies, blue stragglers (BSS: a hotter and bluer extension of normal main sequence stars) represent the highest manifestation of the interplay between stellar evolution and stellar dynamics (Meylan \& Heggie 1997). The origin of BSS is sought as either primordial binaries coeval with the cluster formation epoch, or to a continuous production (in successive epochs) of collisional binaries due to dynamical collisions/encounters experienced by single/binary stars throughout the life of the cluster. Ever since their identification in Sandage (1953), BSS have been subject of many photometric and a handful of spectroscopic (Ferraro et al. 2006a, and references therein) studies. Nevertheless, BSS remain quite difficult to understand in the light of a single comprehensive scenario. Indeed, it is sometimes necessary to invoke both the primordial and collisional mechanisms to explain the BSS distribution in the same cluster. For example the bimodal distribution of BSS in 47Tuc (Ferraro et al. 2004; Mappelli et al. 2004, 2006; highly peaked in the cluster center, rapidly decreasing at intermediate radii, and finally rising again at larger radii) can be interpreted as evidence of two formation scenarios at work: primordial binaries at the cluster periphery (where it is easier for

\footnotetext{
* Based on archival ESO and HST data.
}

them to survive) and collisional binaries at the center (where it is easier to form).

A spectroscopic survey by Preston \& Sneden (2000) of Milky Way field blue metal-poor stars suggested that over $60 \%$ of their sample is made up by binaries, and that at least $50 \%$ of their blue metal-poor sample are BSS. Piotto et al. (2004) presented a homogeneous compilation of $~ 3000$ BSS (based on HST observations of 56 globular cluster), and derived a significant and rather puzzling anti-correlation between the BSS specific frequency and the cluster total absolute luminosity (mass). That is to say that more massive clusters are surprisingly BSS deficient, as if their higher collision rate had no correlation with the production of collisional BSS. Another puzzling observable is that the BSS frequency in Milky Way (MW) field is at least an order of magnitude larger than that of globular clusters. Recently De Marchi et al. (2006) presented a photometric compilation for Galactic open clusters with $-6 \leq M_{V} \leq-3$, and confirmed an extension of the BSS frequency- $M_{V}$ anti-correlation to the open clusters regime. In an attempt to explain these observational trends, Davies et al. (2004) envisage that while the number of BSS produced via collisions tends to increase with cluster mass, becoming the dominant formation channel for clusters with $M_{\mathrm{V}} \leq-8.8$, the BSS number originating from primordial binaries should decrease with increasing cluster mass. 
Accounting for these two opposite trends and binary evolution, Davies et al. (2004) models are able to re-produce the observed BSS population, whose total number seems independent of the cluster mass.

Color-magnitude diagrams (CMD) of typically old dwarf spheroidal galaxies like Ursa Minor (Feltzing et al. 1999; Wyse et al. 2002) show the presence of a well-separated blue plume of stars that very much resembles an old BSS population, as that observed in globular and open clusters. However, in the context of dwarf galaxies one cannot exclude that blue plume stars may include genuinely young main sequence (MS) stars, i.e. a residual star forming activity (e.g. Held 2005, and references therein). The BSS-young MS ambiguity is hard to resolve, and has been discussed before for Carina (Hurley-Keller et al. 1998), Draco (Aparicio et al. 2001), and Ursa Minor (Carrera et al. 2002).

In order to investigate this ambiguity, in this paper we measure the BSS frequency in the dwarf spheroidal galaxy Leo II and collect BSS counts in 8 other galaxies. Dwarf spheroidals/irregulars in which there is current or recent ( $\leq 500 \mathrm{Myr}$ ) star formation are not considered. For example, the Fornax dwarf is known to possess a young population of 200 Myr (e.g. Saviane et al. 2000) with young MS stars brighter than the horizontal branch (HB) level. Therefore Fornax cannot be considered in this paper. The Sagittarius dwarf spheroidal, on the other hand, shows an extended blue plume, yet it does not exceed the HB level, and is included in the study. We also consider the case of the Carina dwarf and re-derive its BSS frequency. As discussed in Hurley-Keller et al. (1998) and Monelli et al. (2003), Carina shows evidence of star formation in recent epochs $(\sim 1 \mathrm{Gyr})$. However, Carina represents the first case in which the MS-BSS ambiguity in dwarf galaxies has been addressed: a BSS frequency was derived and compared with that in Galactic globular clusters. It is also a case in which the "youngest MS stars" do not exceed the HB luminosity level; i.e. Carina does not possess a significant recent star formation rate. As we shall see in Sect. 3.1, the Carina BSS frequency will be most useful for comparison with other dwarf galaxies of similar luminosities.

The BSS frequency is therefore collected for Sagittarius, Sculptor, Leo II, Sextans, Ursa Minor, Draco, Carina, Ursa Major and Boötes and these compared internally, and externally (with that reported for Galactic halo, open and globular clusters). This allows us to address the dependence of the BSS frequency on environment from a wider perspective.

\section{BSS frequency data points}

The dwarf galaxies we study in this paper span a large range of distances ( $\sim 25 \mathrm{kpc}$ for Sagittarius to $\sim 200 \mathrm{kpc}$ for Leo II). This basically precludes the availability of one homogeneous and large-area imaging data-set reaching 1-2 mag below the old MS turn-offs (see however the recent HST/WFPC2 archival survey of dwarf galaxies by Holtzman et al. 2006). Thus estimating the BSS frequency for dwarf galaxies, unfortunately, must rely on a compilation from various sources. We present new reductions of archival imaging from ESO/NTT and HST/WFPC2 for Leo II, and ESO/2.2 $\mathrm{m} \mathrm{BVI} \mathrm{Pre-Flames} 1^{\circ} \times 1^{\circ} \mathrm{WFI}$ mosaic for Sagittarius ${ }^{1}$. These were reduced and calibrated following the standard recipes in Held et al. (1999) and Momany et al. (2001) and (2002). For the remaining dwarf galaxies we estimate the BSS frequency from either public photometric catalogs (Sextans

\footnotetext{
${ }^{1}$ Excluding the inner 14! $\times 14$. region around M 54.
}

by Lee et al. 2003) or published photometry kindly provided by the authors (Ursa Minor by Carrera et al. 2002; Draco by Aparicio et al. 2001; Sculptor by Rizzi et al. 2003; Ursa Major by Willman et al. 2005; Boötes by Belokurov et al. 2006; and Carina by Monelli et al. 2003). All the photometric catalogs extend to and beyond the galaxy half light radius; i.e. we cover a significant fraction of the galaxies and therefore the estimated BSS frequency should not be affected by specific spatial gradients, if present. The only exception is that relative to Sagittarius. With a core radius of $\sim 3.7^{\circ}$, the estimated BSS frequency of our $1^{\circ}$ square degree field refers to less than $3.5 \%$ areal coverage of Sagittarius, or a conservative $\sim 6 \%$ of the stellar populations. Nevertheless, our Sagittarius catalog is one among very few wide-field available catalogs of Sagittarius that reach the BSS magnitude level with an appropriate completeness level, and it is worthwhile to employ it in this BSS analysis.

A delicate aspect of performing star counts is estimating the Galactic foreground/background contribution in the covered area. To compute it in a homogeneous way we made use of the TRILEGAL code (Girardi et al. 2005) that provides synthetic stellar photometry of the Milky Way components (disk, halo, and bulge). Star counts were performed on the simulated diagrams (using the same selection boxes) and subtracted from the observed HB and BSS star counts. We calculate the specific frequency of BSS (normalizing the number of BSS to the HB) as: $F_{\mathrm{HB}}^{\mathrm{BSS}}=\log \left(N_{\mathrm{BSS}} / N_{\mathrm{HB}}\right)$. We remind the reader that uncertainties in the (i) photometric incompleteness correction, (ii) foreground/background subtraction, (iii) possible overlap between old and intermediate age stellar population around the HB level, and (iv) confusion between BSS and normal MS stars, are all unavoidable problems and affect the present and similar studies. The reported error bars account for the propagation of the Poisson errors on the star counts, but mostly reflect the dependence on the uncertainty in properly defining the HB and BSS selection boxes.

\subsection{Dwarf galaxies with a non-standard BSS population}

The Leo II dwarf might be a prototype of a galaxy whose blue plume properties differ from the classical BSS sequence found in globular clusters. Figure 1 displays the HST/NTT CMDs of Leo II upon which we highlight the BSS and HB selection boxes. The Leo II BSS specific frequency was estimated from the HST diagram, whose coverage is comparable with the galaxy half light radius, about $\sim 2$ arcmin. The most notable feature is the detection of a vertical extension in correspondence of the red $\mathrm{HB}$ region. Stars forming this sequence are usually called vertical clump stars (VC, see Gallart et al. 2005). These are heliumburning stars of few hundred Myr to $\sim 1$ Gyr old population whose progenitors are to be searched in the blue plume. Indeed, the relatively large area covered by the NTT shows a CMD with a well defined VC sequence that can be matched by $\sim 1 \mathrm{Gyr}$ isochrones. Since in the context of dwarf galaxies one cannot exclude the presence of an extended star formation, the detection of VC stars (as is also the case for Draco, see Aparicio et al. 2001) would suggest that the blue plume population may well hide a genuinely young main sequence. This possibility will be further investigated in a detailed reconstruction of the star formation history of Leo II (Rizzi et al., in prep.).

A second diagnostic in support of a non-standard BSS sequence lies in the very extension of the Leo II blue plume. The luminosity function of blue stragglers in globular clusters has been found to increase from a luminosity cutoff at $M_{V} \sim 1.9$ toward the ancient MS turn-off at $M_{V} \sim 4.0$ 


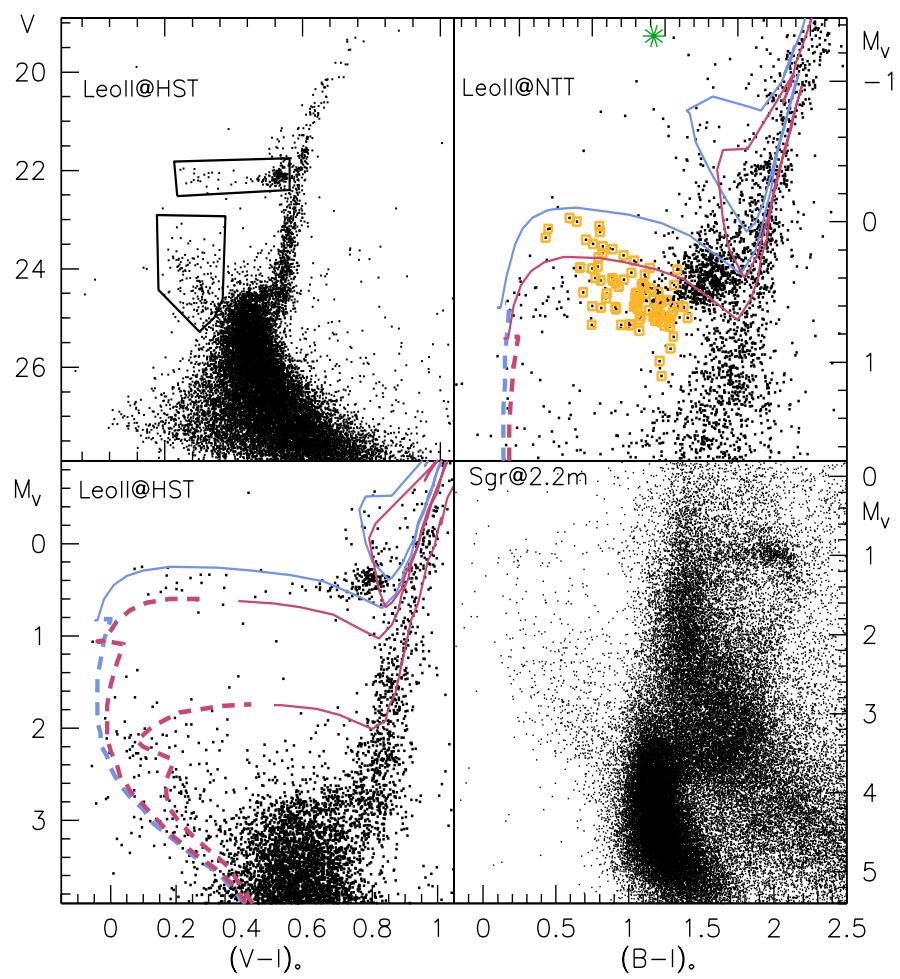

Fig. 1. Left panels display the HST CMDs of Leo II, upon which we highlight the BSS and HB selection boxes and 2.5, 1.2 and $1 \mathrm{Gyr}$, $[\mathrm{Fe} / \mathrm{H}]=-1.3$ isochrones from Girardi et al. (2002). Right upper panel displays the ESO/NTT diagram of Leo II along with 1.0 and $0.8 \mathrm{Gyr}$ isochrones highlighting the extension of the vertical clump sequence (dashed lines mark the MS phase while continuous lines track the postMS evolution). Also plotted are the RR Lyrae stars from Siegel \& Majewski (2000) and one (asterisk) of the 4 anomalous Cepheids (the remaining 3 are outside the NTT field). Lower right panel displays the $\mathrm{ESO} / 2.2 \mathrm{~m}$ CMD of Sagittarius highlighting the Galactic contamination and the extension of its blue plume.

(Sarajedini \& Da Costa 1991; Fusi Pecci et al. 1992). In the case of Leo II, the cutoff luminosity should correspond to $V \simeq 23.8$, whereas we observe it at brighter magnitudes $(V \simeq 23.0$ and $V \simeq 22.7$ in the HST and NTT diagrams respectively), i.e. the BSS sequence in Leo II is more extended than that in globulars.

A third diagnostic is that relative to the ratio of BSS to blue HB stars $\left(7000 \leq T_{\text {eff }} \leq 11500 \mathrm{~K}\right.$ : the lower temperature limit marks the blue border of the RR Lyrae instability strip while the upper temperature limit signs the horizontal branch truncation in dwarf spheroidal galaxies, as noted in Momany et al. 2004). This diagnostic has been used by Hurley-Keller et al. (1998) in favor of a genuine $\sim 1$ Gyr MS population in Carina, and similarly can be applied for Leo II. Basically, for Galactic globular clusters Preston et al. (1994) find a BSS to blue HB ratio of $\sim 0.6$. This is however much lower than what we estimate for Leo II: using the 4 HST/WFPC2 catalogs we find a BSS to blue HB ratio of $\sim 9.2$. A fourth diagnostic is the BSS to $\mathrm{VC}$ ratio that we estimate to remain constant $(\sim 8.1)$ for each of the 4 WFPC 2 chips, as well as for the the entire WFPC2 catalog. This particular observable does not necessarily unveil the true nature of the blue plume in Leo II, it however confirms a tight correlation between the BSS and VC populations.

Lastly, we recall the discovery of four intermediate age anomalous Cepheids in Leo II (Siegel \& Majewski 2000). These are explained as due to either extremely low metallicity variables or to mass transfer (and possibly coalescence) in a close binary systems. However, as discussed by Siegel \& Majewski (2000), should the anomalous Cepheids in Leo II be due to mass transfer binaries, then the number of blue stragglers (in the HST field) is predicted to be $\sim 0.5-5$. This is much lower than the observed number of BSS: corrected for incompleteness, we estimate a total of 175 BSS stars in the HST CMD. Thus, accounting for: (i) the presence of VC stars; (ii) the extension and (iii) number of the BSS population in Leo II makes it more likely that Leo II has experienced an extended star formation history. Similar conclusions can be applied to Sagittarius (possessing an extended blue plume whose cutoff luminosity is brighter than $M_{V} \sim 1.9$, as illustrated in Fig. 1) and Draco (possessing VC stars, as discussed in Aparicio et al. 2001).

Despite all these arguments, it remains difficult to rule out the BSS interpretation. In this regard, it is important to note that the strongest evidence put in favor of a recent star formation episode in Leo II (i.e. the detection of VC stars) is a double-edge sword. Indeed, VC stars have been detected in globular clusters and investigators needed not to invoke a recent star formation in these systems: the presence of the VC sequence could and has been interpreted as due to evolved-BSS. As an example, we consider the case of M80. Ferraro et al. (1999) derive a ratio of the BSS to evolved-BSS (or VC) of $\sim 7$. This is very close to the BSS to VC ratio of $\sim 8$ that we estimate in Leo II. Mighell \& Rich (1996) were the first to suggest that the VC sequence in Leo II can be due to the evolved BSS in the helium-burning phase. Should this be the case then one need not to explain the absence of gas (Blitz \& Robishaw 2000) fueling a recent star formation in Leo II; since there should not be any.

\subsection{Dwarf galaxies showing a standard BSS population}

For few dwarf galaxies there is no hint for the presence of VC stars (see the CMDs of Sextans by Lee et al. 2003 and Ursa Minor by Carrera et al. 2002). Although foreground contamination might contribute in veiling the $\mathrm{VC}$ population, in general, the absence of VC or other intermediate age indicators seemed to indicate a rather normal BSS population in these galaxies.

One interesting anomaly, however, has been detected in the spatial distribution of the BSS population in Sextans. Lee et al. (2003) find that brighter BSS in Sextans are more strongly concentrated towards the galaxy center, while fainter BSS are lacking in the central regions and follow the distribution of old stars in the outer regions. In the context of globular clusters a similar trend is often attributed to the higher occurrence of collisional binaries in higher density environments (i.e. the center) normally producing brighter and bluer BSS. However, the collisional rate in dwarf spheroidals like Sextans is much lower than that in globular clusters (see Sect. 4), and therefore dynamical evolution in Sextans cannot account for a higher production of collisional binaries. Thus, leaving aside this particular distribution anomaly, the overall blue plume properties in galaxies like Sextans, Ursa Minor, Ursa Major and Boötes have been interpreted as the old BSS population.

\section{BSS frequency: analysis}

\subsection{BSS frequency in dwarf galaxies and globular clusters}

We now address the BSS frequency for our dwarf galaxies sample and make an internal comparison. For a wider perspective, we also compare the overall BSS frequency in dwarf galaxies with that observed in other stellar systems. 


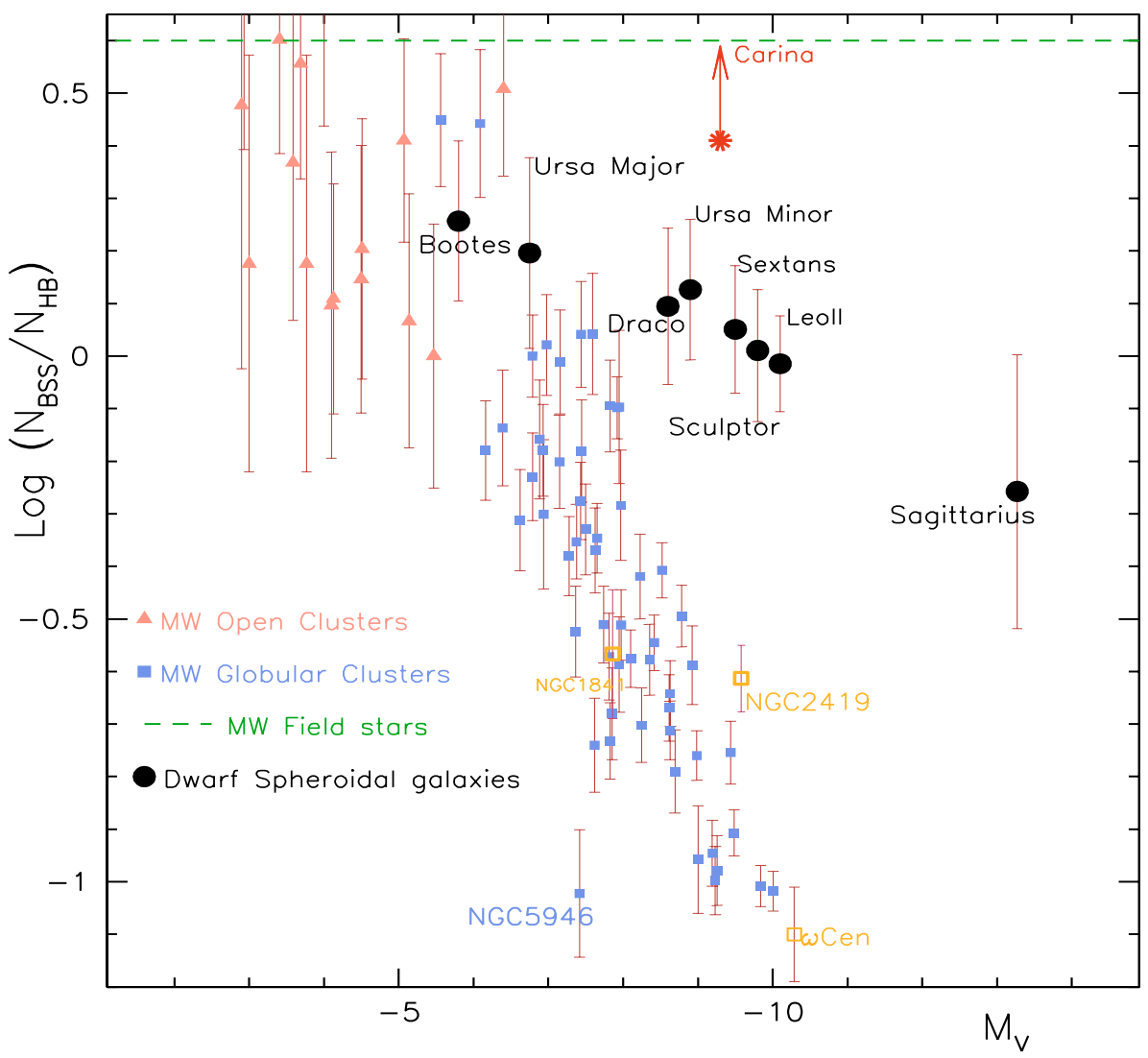

Fig. 2. The $F_{\text {BSS }}$ vs. $M_{V}$ diagram for globular clusters (Piotto et al. 2004) open clusters (De Marchi et al. 2006) and dwarf spheroidal galaxies. The horizontal line shows the mean BSS frequency for Milky Way field stars (Preston \& Sneden 2000).
Figure 2 displays the $F_{\mathrm{BSS}}$ vs. $M_{V}$ diagram for our dwarf galaxy sample together with the data-points of Piotto et al. (2004) and De Marchi et al. (2006) for globular and open clusters, respectively. Of the original open cluster sample we only plot clusters for which $\geq 2$ BSS stars were found. To the globular cluster sample we add the BSS frequency of $\omega$ Cen (as derived by Ferraro et al. 2006b). The anomalies in $\omega$ Cen are multifold and span a multiple MS, sub-giant and red giant branches (see Bedin et al. 2004) and peculiar chemical enrichment (Piotto et al. 2005), that are often used in favor of a dwarf galaxy origin. Moreover we estimate the BSS frequency in 2 peculiar systems: (i) NGC 1841 (Saviane et al. 2003) the LMC most metal-poor and most distant ( $\sim 10 \mathrm{kpc}$ from the LMC bar) globular cluster that is also young and incompatible with the LMC halo rotation; and (ii) NGC 2419 (Momany et al., in prep.) a massive MW cluster at $\sim 90 \mathrm{kpc}$ from the Galactic center. The three datapoints are based on deep HST/ACS, WFPC2 and ACS archival data, respectively. Figure 2 clearly shows that, regardless of their specific peculiarities, $\omega$ Cen, NGC 1841 and NGC 2419 are consistent with the general globular clusters $F_{\mathrm{HB}}^{\mathrm{BSS}}-M_{V}$ anti-correlation.

Before turning our attention to the BSS frequency in dwarf galaxies, we first comment on the case of NGC 6717 and NGC 6838 (the two faintest globular clusters with the highest $F_{\mathrm{HB}}^{\mathrm{BSS}}$ frequency). Located at $(l, b)=\left(13^{\circ},-11^{\circ}\right)$ and $\left(50^{\circ},-5^{\circ}\right)$, the two globular clusters can be subject to significant bulge/disk contamination that was not accounted for in the Piotto et al. analysis. TRILEGAL simulations showed in fact that a considerable number of Galactic young MS stars would overlap with the clusters BSS sequences and this can account for their rather high BSS frequency with respect to globulars of similar $M_{V}$.

Allowing for the exclusion of NGC 6717 and NGC 6838, it results immediately that the lowest luminosity dwarfs (Boötes and Ursa Major) would possess a higher $F_{\mathrm{HB}}^{\mathrm{BSS}}$ than globular clusters with similar $M_{V}$. Most interestingly, their $F_{\mathrm{HB}}^{\mathrm{BSS}}$ is in fact fully compatible with that observed in open clusters. This compatibility between dwarf galaxies and open clusters may suggest that there exists a "saturation" in the BSS frequency (at 0.3-0.4) for the lowest luminosity systems. Thus, the relatively high $F_{\mathrm{HB}}^{\mathrm{BSS}}$ of Boötes and Ursa Major adds more evidence in favor of a dwarf galaxy classification of the 2 systems. Indeed, although their luminosities is several times fainter than Draco or Ursa Minor, the physical size of the two galaxies $\left(r_{1 / 2} \simeq 220\right.$ and 250 pc respectively) exceeds that of more massive galaxies like Ursa Minor $\left(r_{1 / 2} \simeq 150 \mathrm{pc}\right)$.

Another interesting feature is the significant difference between the BSS frequency of Carina with that derived for dwarf galaxies with similar luminosity, i.e. Draco, Ursa Minor, Sextans, Sculptor and Leo II. Although it is only a lower limit ${ }^{2}$, the "BSS" frequency for Carina is of great help in suggesting a threshold near which a galaxy BSS frequency might hide some level of recent star formation. The aforementioned 5 galaxies however have a lower BSS frequency, a hint that these galaxies possess a normal BSS population rather than a young MS. This confirms previous conclusions for Sextans (Lee et al. 2003) and Ursa Minor (Carrera et al. 2002), but is in contradiction with that of Aparicio et al. (2001) for Draco. However, the Aparicio et al. conclusion was mainly based on the detection of the VC stars, a feature that, as we argued, remains an ambiguous indicator. Indeed, Fig. 2 shows that the BSS frequency in Draco is very close to that of Ursa Minor, a galaxy acceptably known to possess an old BSS population.

Lastly, leaving aside the extreme dynamical history of Sagittarius and allowing for the uncertainties (due to the heavy

${ }^{2}$ It is hard to account for the BSS population originating from the older and fainter MS turn-off. 
Galactic contamination and the relatively small sampled populations) it turns out that its blue plume-HB frequency is (i) lower than that of a recently star-forming galaxy like Carina, and most interestingly; (ii) in good agreement with the expected BSS frequency as derived from the $F_{\mathrm{HB}}^{\mathrm{BSS}}-M_{V}$ anti-correlation for the 7 remaining galaxies in our sample. Added to the clear absence of MS stars overlapping or exceeding the Sagittarius HB luminosity level (see Fig. 1), we suggest that the Sagittarius blue plume is a "normal" BSS sequence. As a matter of fact, Sagittarius is probably the nearest system with the largest BSS population: over $2600 \mathrm{BSS}$ stars in the inner $1^{\circ} \times 1^{\circ}$ field.

To summarize, from Fig. 2 one finds that $F_{\mathrm{HB}}^{\mathrm{BSS}}$ in dwarf galaxies is (i) always higher than that in globular clusters, (ii) very close, for the lowest luminosity dwarfs, to that observed in the MW field and open clusters, (iii) the Carina specific $F_{\mathrm{HB}}^{\mathrm{BSS}}$ frequency probably sets a threshold for star-forming galaxies, and most interestingly, (iv) shows a hint of a $F_{\mathrm{HB}}^{\mathrm{BSS}}-M_{V}$ anti-correlation.

\section{2. $A F_{\mathrm{HB}}^{\mathrm{BSS}}-M_{V}$ anti-correlation for dwarf galaxies?}

We here explore the statistical significance of a possible $F_{\mathrm{HB}}^{\mathrm{BSS}}-$ $M_{\mathrm{V}}$ correlation. The linear-correlation coefficient (Bevington 1969) for the 8 galaxies (excluding Carina) data-points is 0.984 . The corresponding probability that any random sample of uncorrelated experimental data-points would yield a correlation coefficient of 0.984 is $<10^{-6}$. Given the greater uncertainties associated with the Sagittarius BSS frequency, one may be interested in the correlation coefficient excluding the Sagittarius data-point. In this case, the resulting correlation coefficient remains however quite high (0.972) and the probability that the 7 remaining data-points would randomly correlate is as low as $10^{-4}$. Thus, the statistical significance of the $F_{\mathrm{HB}}^{\mathrm{BSS}}-M_{V}$ anticorrelation in non star-forming dwarf galaxies is quite high. We follow the methods outlined in Feigelson \& Babu (1992) and fit least-squares linear regressions. In particular, the intercept and slope regression coefficients were estimated through 5 linear models (see the code of Feigelson \& Babu for details) the average of which gives $(a, b)=(0.699 \pm 0.081,0.070 \pm 0.010)$ and $(a, b)=(0.631 \pm 0.120,0.062 \pm 0.014)$ including and excluding the Sagittarius data-point, respectively. The reported errors were estimated through BOOTSTRAP and JACKNIFE simulations so as to provide more realistic $a$ and $b$ errors.

However, to firmly establish this $F_{\mathrm{HB}}^{\mathrm{BSS}}-M_{V}$ anti-correlation one needs to increase the dwarf galaxies sample, in particular at the two luminosity extremes. Unfortunately there are not many non star-forming dwarf galaxies with $-13.3 \leq M_{V} \leq-10.1$ (cf. Table 14 in Mateo 1998), and few exceptions may come from deeper imaging of galaxies like And I and And II. On the other hand, more Local Group dwarf galaxies are being discovered in the low luminosity regime $\left(-8.0 \leq M_{V} \leq-5.0\right)$. Deeper imaging of recently discovered galaxies like Com, CVn IIm, Her and Leo IV (Belokurov et al. 2007) and Willman 1 (Willman et al. 2006) are needed to estimate their BSS frequency. The importance of these low-luminosity galaxies is easily understood once we exclude Boötes and Ursa Major from the BSS frequency correlation analysis. In this case, the correlation coefficient for the 5 remaining data-points is found to drop to 0.901 having a probability of random correlation as high as $1.5 \times 10^{-1}$. Thus, a final word on the $F_{\mathrm{HB}}^{\mathrm{BSS}}-M_{V}$ anti-correlation must await for more data-points at both luminosity extremes.

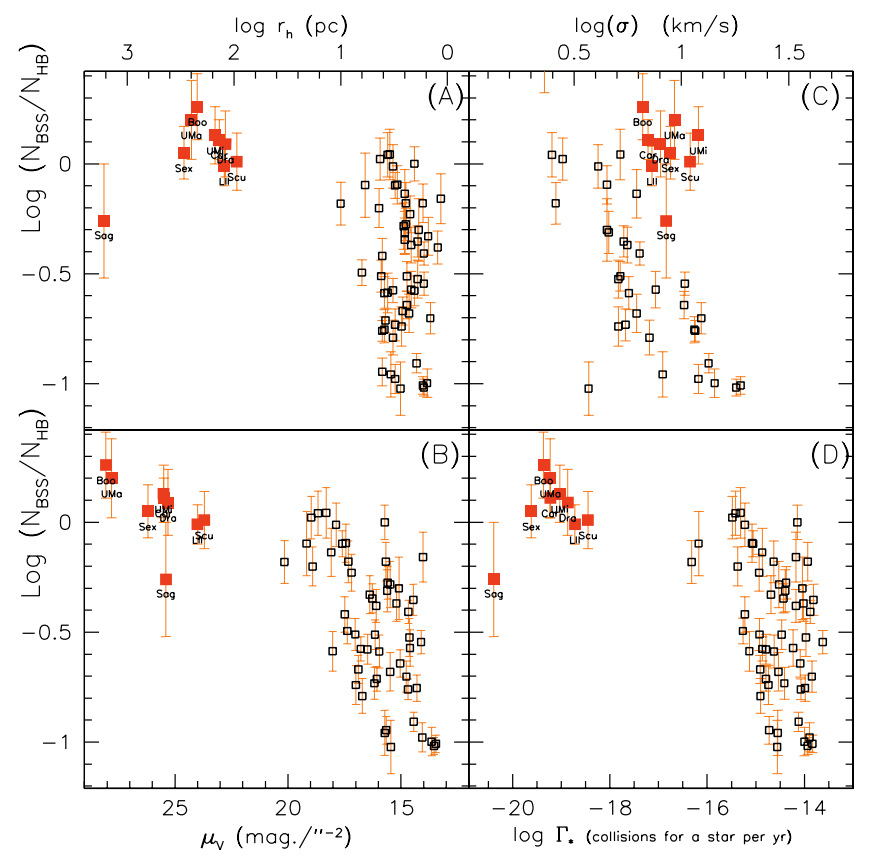

Fig. 3. The BSS frequency as a function of the half light radius (panel a), the central surface brightness (panel b), the velocity dispersion (panel c) and the stellar collision factor (panel d). See text for details.

\section{Discussion and conclusions}

For a sample of 8 non star-forming dwarf galaxies, we have tested the hypothesis that the blue plume populations are made of a genuine BSS population (as that observed in open and globular clusters) and estimated their frequency with respect to HB stars. Should this assumption be incorrect (and the blue plume population is made of young MS stars) then one would not expect an anti-correlation between the galaxies total luminosity (mass) and the blue plume frequency, but rather a correlation between the two. Instead, and within the limits of this and similar analysis, we detect a statistically significant anti-correlation between $F_{\mathrm{HB}}^{\mathrm{BSS}}$ and $M_{V}$. A similar anti-correlation has been reported for Galactic open and globular clusters.

A positive detection of vertical clump stars does not provide a clear-cut evidence in favor of a recent star formation episode in a dwarf galaxy. This is because the vertical clump population has been detected in globular clusters and one cannot exclude that these are evolved-BSS. Thus, the main difference between the blue plume (in non star-forming dwarf galaxies) and the BSS sequence (in globulars clusters) is that regarding their number. Should a dwarf galaxy "obey" the $F_{\mathrm{HB}}^{\mathrm{BSS}}-M_{V}$ anti-correlation displayed by our sample then its blue plume population is probably made of blue stragglers.

Do dwarf galaxies harbor a significant population of collisional binaries? The answer is no. This relies on the intrinsic properties of dwarf galaxies and the consequent difference with those of globular clusters. Indeed, it is enough to recall that the central luminosity density of a dwarf galaxy (e.g. Ursa Minor: $0.006 L_{\odot} \mathrm{pc}^{-3}$ at $\left.M_{V}=-8.9\right)$ is several orders of magnitudes lower than that found in a typical globular cluster (e.g. NGC $7089 \sim 8000 L_{\odot} \mathrm{pc}^{-3}$ at $M_{V}=-9.0$ ). This implies that the collisional parameter of dwarf galaxies is very low, and unambiguously point to the much slower dynamical evolution of dwarf galaxies. To further emphasize this last point, we search for $F_{\mathrm{HB}}^{\mathrm{BSS}}$ dependencies on other dwarf galaxies structural 
parameters. Figure 3 plots the BSS frequency as a function of the half light radius (panel $a$ ), the central surface brightness (panel $b$ ), the velocity dispersion (panel $c$ ) and the stellar collision factor (panel $d)^{3}$. Panels $a$ to $c$ plot the $F_{\mathrm{HB}}^{\mathrm{BSS}}$ as a function of observed globular/dwarf galaxies quantities. Panel $a$ shows that globular clusters and dwarf spheroidals form 2 quite distinct families. This is further confirmed in panel $b$, although the central surface brightness distribution might suggest a $F_{\mathrm{HB}}^{\mathrm{BSS}}$ connection of the two. Panel $c$ shows a correlation between $F_{\mathrm{HB}}^{\mathrm{BSS}}$ and the central velocity dispersion for globular clusters. This reflects the known globular cluster fundamental plane relations, as shown by Djorgovski (1995). Despite the similarities in their velocity dispersion, dwarf galaxies form a separate group from globular clusters, showing systematically higher $F_{\mathrm{HB}}^{\mathrm{BSS}}$.

In panel $d$ we show $F_{\mathrm{HB}}^{\mathrm{BSS}}$ as a function of a calculated quantity: the stellar specific collision parameter $\left(\log \Gamma_{\star}\right.$ : the number of collisions per star per year). More specifically, following Piotto et al. (2004), for both globular clusters and dwarf galaxies we estimate $\log \Gamma_{\star}$ from the central surface density and the system core size. The mean collisional parameter of the 9 studied galaxies is $\simeq-19$. The lowest value is that for Sagittarius with $\log \Gamma_{\star} \simeq-20.2$, and this is due to its very extended galaxy core. Compared with the mean value of -14.8 for the globular clusters sample (see also the lower panel of Fig. 1 in Piotto et al. 2004), the estimated number of collisions per star per year in a dwarf spheroidal is $10^{-5}$ times lower. This almost precludes the occurrence of collisional binaries in dwarf galaxies, and one may conclude that genuine BSS sequences in dwarf galaxies are mainly made of primordial binaries.

Not all primordial binaries, now present in a dwarf galaxy, turn into or are already in the form of BSS. In particular, it is the low exchange encounter probabilities in environments like the Galactic halo or dwarf galaxies that guarantees a friendly environment and a slower consumption/evolution of primordial binary systems. The BSS production (via evolution off the MS of the primary and the consequent mass-transfer to the secondary that may become a BSS) is still taking place in the present epoch and this can explain the high frequency of primordial BSS in dwarf galaxies as well as the Galactic halo.

Lastly, it is interesting to note how the BSS frequency in the low-luminosity dwarfs and open clusters $\left(\log \left(N_{\mathrm{BSS}} / N_{\mathrm{HB}}\right) \sim\right.$ $0.3-0.4)$ is very close to that derived for the Galactic halo $\left(\log \left(N_{\mathrm{BSS}} / N_{\mathrm{HB}}\right) \sim 0.6\right)$ by Preston \& Sneden. The latter value however has been derived relying on a composite sample of only 62 blue metal-poor stars that are (i) distributed at different line of sights; (ii) at different distances; and most importantly, (iii) for which no observational BSS-HB star-by-star correspondence can be established. Thus, allowing for all these uncertainties in the field BSS frequency (see also the discussion in Ferraro et al. 2006b), it is safe to conclude that the observed open clustersdwarf galaxies BSS frequency sets a realistic, and observational upper limit to the primordial BSS frequency in stellar systems.

Acknowledgements. We thank Alvio Renzini and Giampaolo Piotto for useful discussions that helped improve this paper. We are also grateful to Belokurov V., Willman B., Carrera R., Monelli M. and Aparicio A. for kindly providing us their photometric catalogs.

3 For globular clusters we make use of the Trager et al. (1995) and Pryor \& Meylan (1993) tables, whereas for dwarf spheroidals we use the tables from Mateo (1998) and updated velocity dispersions from recent measurements (Sculptor: Tolstoy et al. 2004; Sextans: Walker et al. 2006); Carina: Koch et al. (2007); Boötes: Muñoz et al. (2006a); Ursa Minor: Muñoz et al. (2005); Ursa Major: Kleyna et al. (2005); Sagittarius: Zaggia et al. (2004); Draco: Muñoz et al. (2005); Carina: Muñoz et al. (2006b).

\section{References}

Aparicio, A., Carrera, R., \& Martínez-Delgado, D. 2001, AJ, 122, 2524 Bedin, L. R., Piotto, G., Anderson, J., et al. 2004, ApJ, 605, L125 Belokurov, V., Zucker, D. B., Evans, N. W., et al. 2006, ApJ, 647, L111 Belokurov, V., Zucker, D. B., Evans, N. W., et al. 2007, ApJ, 654, 897 Bevington, P. R. 1969, Data reduction and error analysis for the physical sciences (New York: McGraw-Hill)

Blitz, L., \& Robishaw, T. 2000, ApJ, 541, 675

Carrera, R., Aparicio, A., Martínez-Delgado, D., \& Alonso-García, J. 2002, AJ, 123, 3199

Davies, M. B., Piotto, G., \& de Angeli, F. 2004, MNRAS, 349, 129

de Marchi, F., de Angeli, F., Piotto, G., Carraro, G., \& Davies, M. B. 2006, A\&A, 459,489

Djorgovski, S. 1995, ApJ, 438, L29

Feigelson, E. D., \& Babu, G. J. 1992, ApJ, 397, 55

Feltzing, S., Gilmore, G., \& Wyse, R. F. G. 1999, ApJ, 516, L17

Ferraro, F., Paltrinieri, B., Rood, R. T., \& Dorman, B. 1999, ApJ, 522, 983

Ferraro, F. R., Beccari, G., Rood, R. T., et al. 2004, ApJ, 603, 127

Ferraro, F. R., Sabbi, E., Gratton, R., et al. 2006a, ApJ, 647, L53

Ferraro, F. R., Sollima, A., Rood, R. T., et al. 2006b, ApJ, 638, 433

Fusi Pecci, F., Ferraro, F. R., Corsi, C. E., Cacciari, C., \& Buonanno, R. 1992, AJ, 104, 1831

Gallart, C., Zoccali, M., \& Aparicio, A. 2005, ARA\&A, 43, 387

Girardi, L., Bertelli, G., Bressan, A., et al. 2002, A\&A, 391, 195

Girardi, L., Groenewegen, M. A. T., Hatziminaoglou, E., \& da Costa, L. 2005, A\&A, 436, 895

Held, E. V. 2005, Near-fields cosmology with dwarf elliptical galaxies, IAU Coll., 198, 11

Held, E. V., Saviane, I., \& Momany, Y. 1999, A\&A, 345, 747

Holtzman, J. A., Afonso, C., \& Dolphin, A. 2006, ApJS, 166, 534 WFPC2 Hurley-Keller, D., Mateo, M., \& Nemec, J. 1998, AJ, 115, 1840

Kleyna, J., Wilkinson, M., Evans, N., \& Gilmore, G. 2005, ApJ, 630, L141

Koch, A., Grebel, E., Kleyna, J., et al. 2007, AJ, 133, 270

Lee, M. G., Park, H. S., Park, J.-H., et al. 2003, AJ, 126, 2840

Mateo, M. L. 1998, ARA\&A, 36, 435

Meylan, G., \& Heggie, D. C. 1997, A\&ARv, 8, 1

Mighell, K. J., \& Rich, R. M. 1996, AJ, 111, 777

Mapelli, M., Sigurdsson, S., Colpi, M., et al. 2004, ApJ, 605, L29

Mapelli, M., Sigurdsson, S., Ferraro, F. R., et al. 2006, MNRAS, 373, 361

Mighell, K. J. 1997, AJ, 114, 1458

Monelli, M., Pulone, L., Corsi, C. E., et al. 2003, AJ, 126, 218

Momany, Y., Vandame, B., Zaggia, S., et al. 2001, A\&A, 379, 436

Momany, Y., Held, E. V., Saviane, I., \& Rizzi, L. 2002, A\&A, 384, 393

Momany, Y., Cassisi, S., Piotto, G., et al. 2003, A\&A, 407, 303

Momany, Y., Bedin, L. R., Cassisi, S., et al. 2004, A\&A, 420, 605

Muñoz, R. R., Frinchaboy, P. M., Majewski, S. R., et al. 2005, ApJ, 631, L137

Muñoz, R., Carlin, J., Frinchaboy, P., et al. 2006a, ApJ, 650, L51

Muñoz, R. R., Majewski, S. R., Zaggia, S., et al. 2006b, ApJ, 649, 201

Piotto, G., De Angeli, F., King, I. R., et al. 2004, ApJ, 604, L109

Piotto, G., Villanova, S., Bedin, L. R., et al. 2005, ApJ, 621, 777

Preston, G. W., \& Sneden, C. 2000, AJ, 120, 1014

Preston, G. W., Beers, T. C., \& Shectman, S. A. 1994, AJ, 108, 538

Pryor, C., \& Meylan, G. 1993, ASP Conf. Ser., Structure and Dynamics of Globular Clusters, 50, 357

Rizzi, L., Held, E., Momany, Y., et al. 2003, Mem. Soc. Astron. Ital., 74, 510

Sandage, A. R. 1953, AJ, 58, 61

Sarajedini, A., \& Da Costa, G. S. 1991, AJ, 102, 628

Saviane, I., Held, E. V., \& Bertelli, G. 2000, A\&A, 355, 56

Saviane, I., Rosenberg, A., Aparicio, A., \& Piotto, G. 2003, ASP Conf. Ser., New Horizons in Globular Cluster Astronomy, 296, 402

Siegel, M. H., \& Majewski, S. R. 2000, AJ, 120, 284

Shetrone, M. D., Côté, P., \& Stetson, P. B. 2001, PASP, 113, 1122

Smecker-Hane, T. A., Stetson, P. B., Hesser, J. E., \& Vandenberg, D. A. 1996, From Stars to Galaxies: the Impact of Stellar Physics on Galaxy Evolution, ASP Conf. Ser., 98, 328

Trager, S. C., King, I. R., \& Djorgovski, S. 1995, AJ, 109, 218

Tolstoy, E., Irwin, M. J., Helmi, A., et al. 2004, ApJ, 617, L119

Walker, M. G., Mateo, M., Olszewski, E. W., et al. 2006, ApJ, 642, L41

Willman, B., Dalcanton, J. J., Martinez-Delgado, D., et al. 2005, ApJ, 626, L85

Willman, B., Masjedi, M., Hogg, D. W., et al. 2006 [arXiv: astro-ph/0603486]

Wyse, R., Gilmore, G., Houdashelt, M., et al. 2002, New Astron., 7, 395

Zaggia, S., Bonifacio, P., Bellazzini, M., et al. 2004, Mem. Soc. Astron. It. Suppl., 5, 291 